Sharif University of Technology
Scientia Iranica
SCIENTIA

Invited Paper

\title{
Distribution power system outage diagnosis based on root cause analysis
}

\author{
M.S. Bashkari, A. Sami*, M. Rastegar*, and M.J. Bordbari \\ School of Electrical and Computer Engineering, Department of Engineering, Shiraz University, Zand Avenue, Shiraz, P.O. Box \\ 71348-51154, Iran.
}

Received 7 August 2019; received in revised form 1 September 2019; accepted 7 October 2019

\author{
KEYWORDS \\ Power system \\ reliability; \\ Distribution system \\ outages; \\ Data mining; \\ Random forest; \\ Cost matrix; \\ Ensemble.
}

\begin{abstract}
This paper proposes data-mining-based models to diagnose outage data in distribution power systems. In this work, outage data from a local distribution company was gathered and aligned with weather data. Then, a subset of features was selected to reduce the processing time and simplifying purposes. To increase the fairness of the final models and to account for differences in misclassification cost, a customized cost matrix was used. Two decision-tree-based modeling algorithms were trained and tested. Results showed the ability of the established models to diagnose the root cause of an outage fairly well. In addition, an ensemble of the decision-tree-based models was built, which outperformed the other two models in almost all cases. Finally, applications of such models to decreasing outage duration and improving the reliability of the power distribution network were investigated.
\end{abstract}

(C) 2019 Sharif University of Technology. All rights reserved.

\section{Introduction}

Data mining has been attracting the attention of most researchers in almost every science category as a way to turn the problem of large accumulated data into an opportunity. Data mining methods like classification, clustering, outlier detection, and visualization give the researchers a chance to gain a better understanding of the category. Power system is one of the research areas that has profited from contributions of data mining methods. Meter and power consumption data have been used in several types of research to classify consumers or to identify distinctive ones, hence detecting electricity theft [1-3]. Visualization techniques have been used to ease the monitoring operations [4-5] and

*. Corresponding authors.

E-mail addresses: sami@shirazu.ac.ir (A. Sami); mohammadrastegar@shirazu.ac.ir (M. Rastegar)

doi: $10.24200 /$ sci.2019.54190.3638 to realize sudden changes in the parameters of power systems [6].

In recent years, with the availability of outage data, the literature on reliability of power distribution networks has been augmented. This is due to the fact that most outages in power system originate in the distribution section of the network [7]. Since many types of research show that the value of the lost load is extremely higher than its actual cost [8], studying reliability of the power systems, especially distribution systems, seems inevitable. Reliability of distribution system depends on many parameters, such as number of outage occurrences, duration of the outages, and number of affected consumers. Therefore, investigating the impacts of these parameters and roots, which endanger reliability of distribution system, has recently gained considerable attention. Since various and frequent data are available in this context, data mining methods have been utilized for diagnosis or prognosis of failure occurrences in the system. For example, component outages caused by hurricane were predicted by 
Eskandarpour and Khodaei [9] using logistic regression. Wanik et al. [10] investigated the effect of storm on outages using high-resolution weather simulation data by decision tree, random forest, and boosted gradient tree. Significant correlating factors in outage occurrence were studied by Doostan and Chowdhury [11] using association rule mining and by Cai and Chow [12] using fuzzy inference system. In the study carried out by Chow and Taylor [13], animal-related outages were investigated, several related factors were introduced, and a prevention method was proposed. Other researches have been performed to estimate fault location, hence shortening the investigation part of restoration. For example, Discrete Fourier Transform (DFT) and artificial neural networks were used by Warlyani et al. [14] to determine faulty sections in teed transmission lines. Fault causes are often classified as vegetation-, animal, and weather-related [15] in addition to equipment failure [16] groups. Many researches have tackled the problem of identifying the root cause of an outage. This problem was addressed using fuzzy classification to identify vegetation-, animal-, and lightening-related outages [15] and to determine equipment failure and vegetation-related outages $[16,17]$. All these researches have used historical data from the past outages and utilized multi-label or binary classification methods to identify the main outage cause upon occurring.

In this paper, data-mining-based methods are used to find a predictive model for analyzing and deducing the root cause of the power distribution system outages upon occurrence. To this end, fouryear outage data from a local distribution company and local weather data are integrated. Then, outage cause labels from outage data are reclassified into vegetation-, animal-, equipment-, and weather-related categories. Other fault causes that do not belong to these classes are labeled as 'other.' After reclassifying the outage labels, data are divided into train and test parts. A customized cost matrix is configured based on the importance of each class and the balance ratio between classes. In this case, using a cost matrix is crucial for two reasons. First, the cost of incorrect classification of an outage class should not be the same for all cases. Therefore, some misclassifications should receive higher penalty. Another important reason to use the cost matrix is to account for imbalance ratio of classes, which may result in invaluable realization. After building the cost matrix, several data mining classification models, such as random forest [18] and C5.0 $[19,20]$, are considered for the training part. Then, generalizability of these two models is verified against the test part. In addition, another model is built as an ensemble [21] of the previous ones, which inherits the best characteristics of each model and outperforms both models in most cases. The models are tested and compared to show their ability in predicting the cause of outages upon occurrence. The results are presented and the effects of such predictors on the reliability of distribution system are discussed.

\section{Material and methods}

Data sources used in this work are non-simulated real-world data. Distribution outage data have been collected from a local distribution network, of which the characteristics are presented in Table 1, consisting of substation name, outage cause, date, time of occurrence, and a coded feeder number, among other features. Also, weather data for the area are retrieved from a reliable prognosis weather database [22] for the same period of time. Time and date, temperature, humidity, pressure, precipitation, and wind speed are some of notable features of the data.

\subsection{Preprocessing}

The described, data sources have different temporal granularities. In other words, weather data are collected regularly every three hours, whereas each row of outage data is appended to the data source upon outage occurrence. This fact leads to the generation of two data sources with different timestamps. To resolve this issue, the two data sources are integrated.

More preprocessing steps to make the resulting dataset ready are taken as follows. In the aforementioned distribution company, 96 outage cause labels are used by technicians to classify outage causes. Some of these labels are related and could be combined together and some others could be removed for being either less frequent or less important than others. Therefore, first, scheduled outages are removed from the data since they are inevitable and could be planned to be less effective on reliability. Outages that are self-resolving or the ones whose durations are less than 5 minutes are also removed due to low effect on reliability. Then, the remaining outage causes are reclassified and labeled as vegetation-, animal-, equipment-, and weather-related. Other fault causes that could not be classified in the aforementioned classes are categorized as 'other.' With further inspection of data, some features with more than $95 \%$ null values are removed. Moreover, since online root cause analysis models should be able to work as soon as outage occurs, some other features like Energy Not Supplied (ENS) or restoration time

Table 1. Characteristics of distribution network.

\begin{tabular}{ll}
\hline Substations & 64 \\
Feeders & 491 \\
Feeder length (km) & 10981 \\
Distribution transformers & 26155 \\
Underground posts & 1593 \\
Overhead posts & 23207 \\
\hline
\end{tabular}




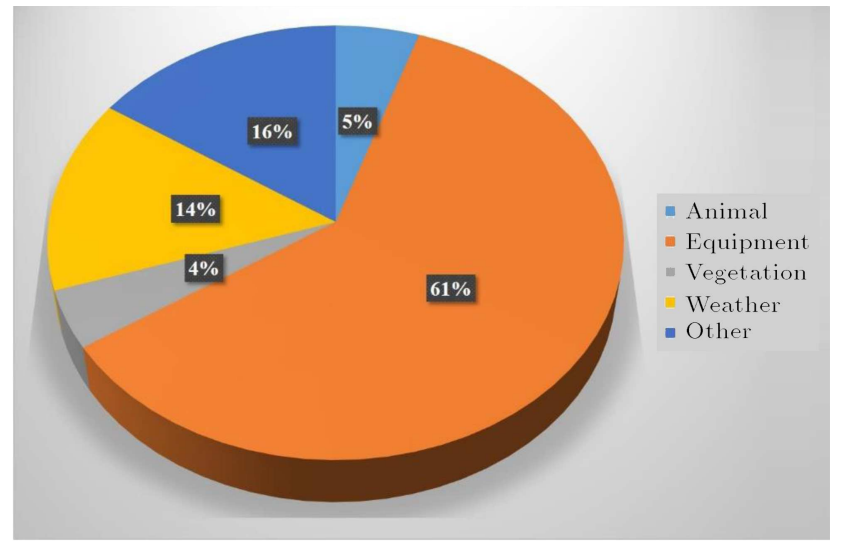

Figure 1. Distribution of root cause classes in dataset.

are removed, because they are not available within 5 minutes from outage manifestation.

\subsection{Visualization}

To get a better understanding of the data, several illustrations of the relations between features are created and investigated. Figure 1 shows the imbalance ratio of class members. As it is illustrated, most of the records in the data are from the equipment class. This would be problematic in modeling phase, because most of the modeling algorithms tend to favor the majority class to improve accuracy. This problem is addressed before training the models.

To investigate the effect of region on each root cause of outages, the number of outages is illustrated in Figure 2 separately for each substation, divided by root cause. It is obvious that outages occur less frequently in some substations such as $16,23,41,44,50$, and 52 . On the other hand, substations 11, 12, 21, 46, 58, and 63 have more outage count than the others. Moreover, the ratio of the root causes in each substation is different. For example, animal caused outages are responsible for a higher rate of outages in substation 12 than in substation 58. This shows that to predict the root cause of an outage, a model should consider substation number as an input.

\subsection{Feature selection}

With the growth of computer power and advancement in data collection technologies, there are considerable data generated in every field. Even using data mining and machine learning algorithms, this amount of data is hard to analyze. Feature selection is one of the methods that deals with this problem. It is the operation to choose a smaller subset from the main set of features without sacrificing accuracy by removing irrelevant or redundant features. This operation allows us to achieve the same performance of predictor or even improve it and, at the same time, increase comprehensibility of the model [23]. In this paper, feature selection is performed in two phases. First, features are ranked based on their correlation to the outage cause. This type of feature selection is called filter method [24]. The correlation could be measured by likelihood ratio or Pearson metrics, among others. In this paper, since the sample size is relatively small, likelihood ratio is preferred. This phase is done regardless of the chosen predictor model and other inputs. Based on quality of the features and diversity of values, 25 features are selected in total.

In the next phase of feature selection, which is similar to wrapper methods [24], predictor model and correlations between input fields are considered. In this

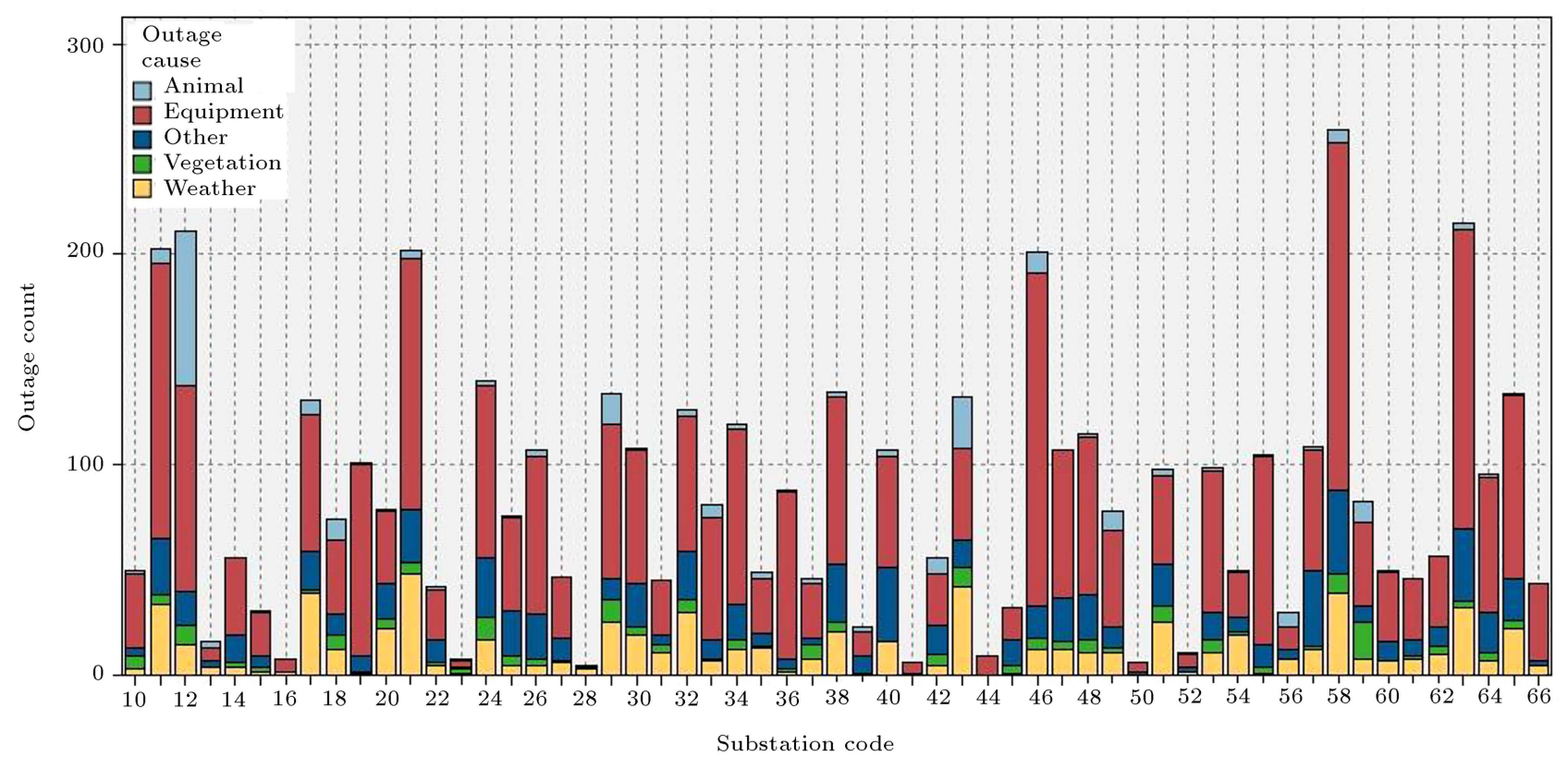

Figure 2. Number of root causes of outages in different substations. 
phase, the subset of features chosen in the filter phase is used as input. Since different models are built, in each model, a different number of features are selected and used in the training phase. Figures 3 and 4 illustrate the most important features based on random forest and C5.0 algorithms, respectively.

\subsection{Cost matrix}

Since misclassification of records is costly, in many data mining algorithms, there is a cost matrix that

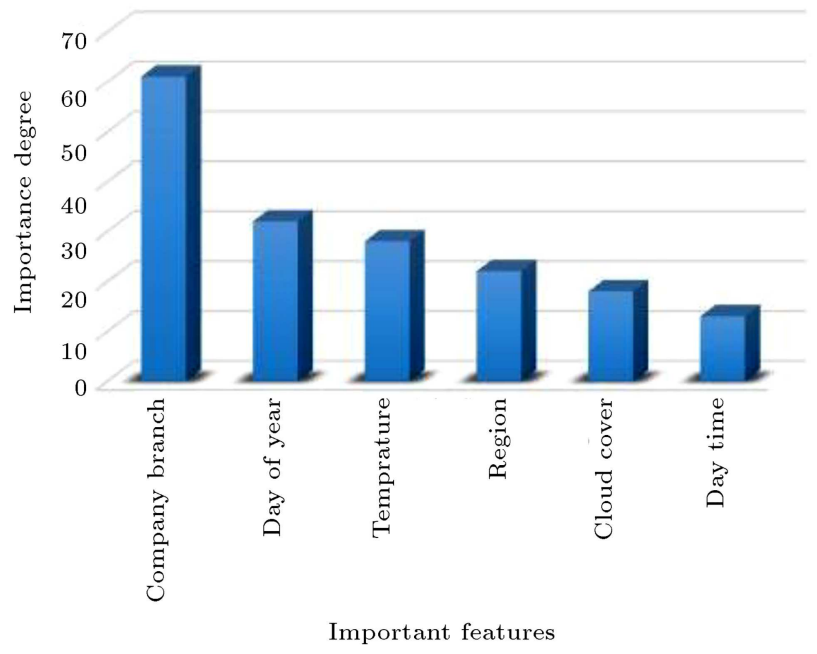

Figure 3. Feature importance for random forest algorithm.

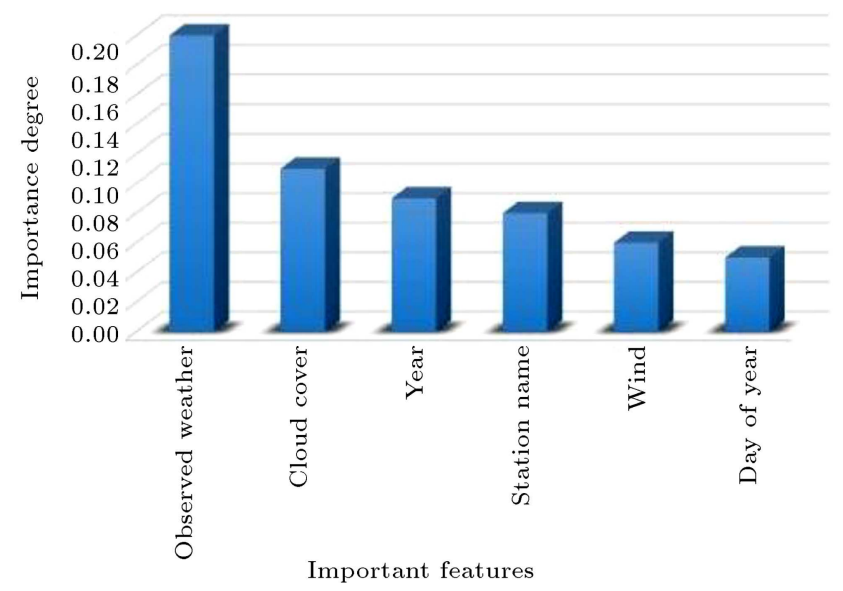

Figure 4. Feature importance for C5.0 algorithm.
Table 2. A common cost matrix.

\begin{tabular}{lcccc}
\hline & & \multicolumn{3}{c}{ Predicted } \\
\cline { 3 - 5 } & & Class 1 & Class 2 & Class 3 \\
\hline \multirow{3}{*}{ Observed } & Class 1 & 0 & 1 & 1 \\
& Class 2 & 1 & 0 & 1 \\
& Class 3 & 1 & 1 & 0 \\
\hline
\end{tabular}

inputs cost of incorrectly classified records based on the observed label and the predicted label. In many use cases, there is not much difference between the costs of misclassifications. In other words, cost is not dependent on the observed and predicted labels and almost all misclassification costs are the same. Table 2 shows a common cost matrix used in many classifiers.

In the cases that the cost of misclassification varies for each observed and predicted pair, the cost matrix can be configured to reflect this difference. For example, in this research, misclassifying the 'equipment' class as 'other' would cost significantly more than misclassifying 'other' as 'equipment.' The reason is that in the first case, the repair crew might be dispatched without being properly prepared to deal with equipment failure and it would prolong the restoration process. On the other hand, being prepared for equipment failure would not significantly influence readiness of the repair crew to resolve other types of failure.

As illustrated in the visualization section, records are not equally distributed in all classes. Rather, more than half of the records are from equipment class. In such imbalanced cases, in order to improve the overall accuracy, data mining algorithms favor the major class over other classes. This would result in accurate models, which may be impractical in real-world situations. Another important use of a customized cost matrix is to deal with this kind of situations by increasing the cost of misclassifying other classes as the major class. Table 3 shows the cost matrix, which is configured to deal with the above issues. As the least important class, the 'other' row has been altered to be zero so that there is no penalty for misclassifying members of this class.

In Table 3, using cost values in equipment failure column same as those in 'other' columns would result

Table 3. Customized cost matrix used in this paper.

\begin{tabular}{llccccc}
\hline & \multicolumn{5}{c}{ Predicted } \\
\cline { 3 - 7 } & Animal & 0 & 2 & 1 & 1 & 1 \\
\hline \multirow{3}{*}{ Observed } & Equipment & 1 & 0 & 1 & 1 & 1 \\
& Other & 0 & 0 & 0 & 0 & 0 \\
& Vegetation & 1 & 2 & 1 & 0 & 1 \\
& Weather & 1 & 2 & 1 & 1 & 0 \\
\hline
\end{tabular}


in a model that predicts most root causes to be of the 'equipment' class. To prevent this issue and to build a fair model, costs in this column have been changed so that predicting animal, vegetation, and weather classes as 'equipment' class is more costly. In this way, the model will adapt to predict classes in a more trustworthy manner.

\subsection{Model training}

The goal of this paper is to build a predictive model to analyze and deduce the root cause of outages upon occurrence. This would greatly help the network operator choose and dispatch the most suited and best-equipped repair crew available to deal with the failure. To this end, two data-mining-based methods and an ensemble method are used, as described in the following.

\subsubsection{Random forest results and discussion}

Random forest algorithm was introduced by Breiman [18]. In the random forest algorithm, a model is built in several iterations. In each iteration, a sample of the data is chosen, which is called a bootstrap sample. Then, a single decision tree is built based on this sample. To this end, at the root node of the tree, the sample data are divided into two subgroups. Subgroups are chosen based on an impurity index. Then, for each new node, the respective subgroup is split into two more subgroups based on the same index. The algorithm continues until a stopping condition is met and a single decision tree is constructed. To build a random forest model, hundreds of decision trees are built in the same way using different bootstrap samples. To predict the class of a new instance, every tree makes a decision between available classes. The final decision of this model is made by voting between all of the trees in the forest. This algorithm is more robust against overfitting [18] in comparison with other decision-tree-based algorithms, as a result of this randomness. Thus, the predictions on the test part could be expected to have the same accuracy as the predictions on the train part. Table 4 presents the results of applying this algorithm to integrated outage and weather data. Each row shows observed or real root cause of outages, while each column indicates the predicted label by random forest algorithm. The number in each cell is the percentage that the members of the representative row class may be labeled by the predictor as the respective column class. Bolded values show the accuracy of the predictor in identifying the respective class. This table shows that random forest algorithm is able to predict animal, equipment, vegetation, and weather classes fairly well. Because of using customized cost matrix in this predictor, none of the records of these four important classes is falsely predicted as 'other,' as predicting 'other' would not help the repair crew to prepare and diagnose the problem. Furthermore, although the equipment class is by far the most populated class, the predictor does not significantly prioritize this class over other classes.

Table 5 shows the results of the model built by

Table 4. Random forest-based predictor with customized cost matrix; observed versus predicted.

\begin{tabular}{llccccc}
\hline & \multicolumn{5}{c}{ Predicted } \\
\cline { 3 - 7 } & & Animal & Equipment & Other & Vegetation & Weather \\
\hline \multirow{3}{*}{ Observed } & Animal & $\mathbf{7 2 . 7 3}$ & 25.76 & 0.00 & 1.52 & 0.00 \\
& Equipment & 7.45 & $\mathbf{8 3 . 2 8}$ & 0.00 & 2.29 & 6.99 \\
& Other & 7.26 & 79.49 & $\mathbf{1 . 2 8}$ & 3.85 & 8.12 \\
& Vegetation & 5.63 & 26.76 & 0.00 & $\mathbf{4 7 . 8 9}$ & 19.72 \\
& Weather & 1.93 & 9.18 & 0.00 & 7.25 & $\mathbf{8 1 . 6 4}$ \\
\hline
\end{tabular}

Table 5. Random forest-based predictor with default cost matrix; observed versus predicted.

\begin{tabular}{llccccc}
\hline & \multicolumn{5}{c}{ Predicted } \\
\cline { 3 - 7 } & & Animal & Equipment & Other & Vegetation & Weather \\
\hline \multirow{3}{*}{ Observed } & Animal & $\mathbf{1 . 2 5}$ & 96.25 & 0.00 & 0.00 & 2.50 \\
& Equipment & 0.36 & $\mathbf{9 6 . 7 9}$ & 0.24 & 0.12 & 2.49 \\
& Other & 0.00 & 87.18 & $\mathbf{5 . 9 8}$ & 1.28 & 5.56 \\
& Vegetation & 0.00 & 61.40 & 5.26 & $\mathbf{1 0 . 5 3}$ & 22.81 \\
& Weather & 0.00 & 30.10 & 1.02 & 1.02 & $\mathbf{6 7 . 8 6}$ \\
\hline
\end{tabular}


the random forest algorithm considering the default cost matrix. As expected, the algorithm optimizes the model for the highest accuracy and, as a result, most instances are predicted to belong to the majority classes, i.e., equipment and weather. While this model improves the accuracy of the equipment class, it immensely reduces accuracy of other classes. Thus, this model may not be applicable to a real-world power distribution network.

\subsubsection{C5.0 results and discussion}

The C5.0 algorithm, proposed in 1997 by JR Quinlan, is based on the basic decision tree and $\mathrm{C} 4.5$ algorithm [20]. Unlike the random forest algorithm, C5.0 provides a single decision tree and does not use bootstrap sampling. The advantage of building a single tree is that the results could be interpreted and used as new knowledge in the field. On the other hand, C5.0 model suffers from overfitting problem, which occurs when a model has high accuracy for the train part and low accuracy for the test part. Hence, the model cannot be generalized well to new instances.

Table 6 shows the results of the built model based on the C5.0 algorithm. Like the random forest algorithm, C5.0 shows promising results in predicting the root cause of outages. The C5.0 model is outperformed by random forest in two important classes, but it has the advantage of being simpler to understand than the random forest model. The reason for the simplicity of the C5.0 model is that it builds a single tree, unlike the random forest-based model, which operates as a black box predictor and does not reveal the logic behind each prediction.

Table 7 shows the results of the model built by using C5.0 algorithm without the customized cost matrix. It shows that, similar to the random forest algorithm, this model predicts most of the instances as equipment and weather, which are the majority classes, while extenuating the other important classes, like animal and vegetation, drastically.

\subsubsection{Ensemble results and discussion}

Ensemble method was proposed in 1993 by Hinton and has been used to combine models to get accurate results. Since none of the presented models is completely dominant over the other, to capture the most confident prediction of each model, using the ensemble of both models is proposed. As it is observed in Tables 4 to 7 , while the random forest algorithm predicts vegetation and weather causes more accurately, in the case of equipment failure, it is outperformed by the C5.0 model. To combine these models, two conditions are considered based on the class predictions for each record. In the case of an agreement between the labels suggested by both algorithms, the suggested label will also be produced as the output of the ensemble model. In the case of a disagreement between output labels of the models, the classifier with the highest confidence will be selected. Based on these circumstances, the ensemble results are generated and presented in Table 8. It is observed that the ensemble model outperforms C5.0 in all cases and it is rather better than the random forest model in all, except for vegetation-related, outages.

To compare the results of C5.0, random forest, and the proposed ensemble algorithms, the accuracy of all presented models for each class is presented in Figure 5. Based on this illustration, the models that

Table 6. C5.0 based predictor with customized cost matrix; observed versus predicted.

\begin{tabular}{llcccc}
\hline & & \multicolumn{4}{c}{ Predicted } \\
\cline { 3 - 6 } & & Animal & Equipment & Vegetation & Weather \\
\hline \multirow{3}{*}{ Observed } & Animal & $\mathbf{7 2 . 7 3}$ & 27.27 & 0.00 & 0.00 \\
& Equipment & 3.09 & $\mathbf{9 0 . 9 5}$ & 0.69 & 5.27 \\
& Other & 4.27 & 81.20 & 2.14 & 12.39 \\
& Vegetation & 9.86 & 43.66 & $\mathbf{2 3 . 9 4}$ & 22.54 \\
& Weather & 1.45 & 17.39 & 2.42 & $\mathbf{7 8 . 7 4}$ \\
\hline
\end{tabular}

Table 7. C5.0 based predictor with default cost matrix; observed versus predicted.

\begin{tabular}{llccccc}
\hline & \multicolumn{5}{c}{ Predicted } \\
\cline { 3 - 7 } & & Animal & Equipment & Other & Vegetation & Weather \\
\hline \multirow{3}{*}{ Observed } & Animal & $\mathbf{2 . 4 4}$ & 95.12 & 0.00 & 0.00 & 2.44 \\
& Equipment & 0.22 & $\mathbf{9 5 . 7 3}$ & 0.66 & 0.11 & 3.29 \\
& Other & 0.00 & 92.48 & $\mathbf{3 . 5 4}$ & 1.33 & 2.65 \\
& Vegetation & 0.00 & 70.00 & 6.67 & $\mathbf{1 5 . 0 0}$ & 8.33 \\
& Weather & 1.82 & 32.73 & 1.36 & 0.91 & $\mathbf{6 3 . 1 8}$ \\
\hline
\end{tabular}


Table 8. Ensemble model output; observed versus predicted.

\begin{tabular}{llcccc}
\hline & & \multicolumn{4}{c}{ Predicted } \\
\cline { 3 - 6 } & & Animal & Equipment & Vegetation & Weather \\
\hline \multirow{3}{*}{ Observed } & $\mathbf{7 4 . 2 4}$ & 25.76 & 0.00 & 0.00 \\
& Equipment & 2.29 & $\mathbf{9 1 . 0 7}$ & 1.03 & 5.61 \\
& Other & 2.56 & 83.33 & 2.14 & 11.97 \\
& Vegetation & 5.63 & 39.44 & $\mathbf{3 9 . 4 4}$ & 15.49 \\
& Weather & 0.00 & 14.01 & 2.42 & $\mathbf{8 3 . 5 7}$ \\
\hline
\end{tabular}

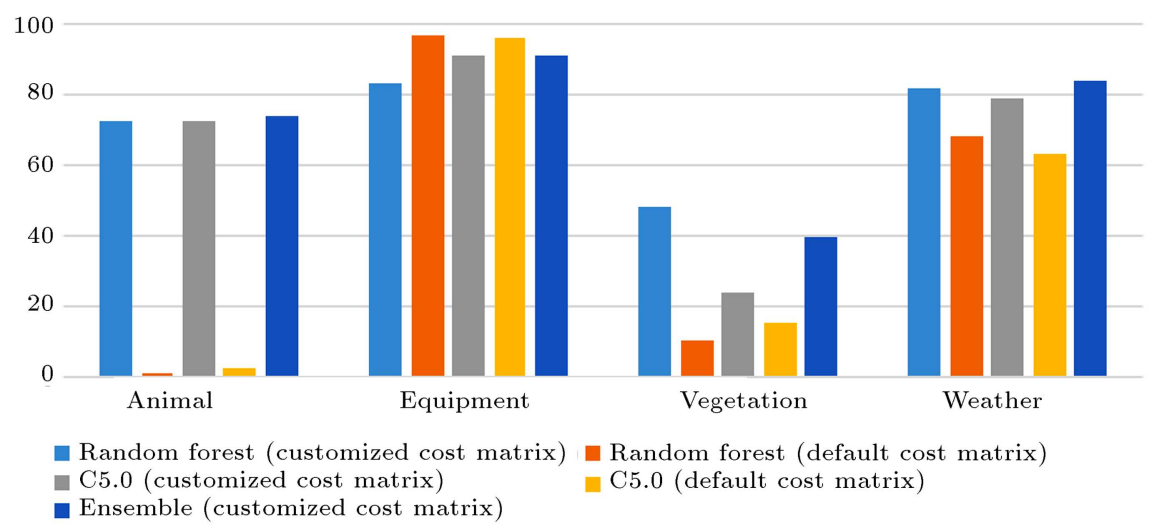

Figure 5. Comparison of the accuracy of predictors.

use default cost matrices fail to predict animal and vegetation classes and have the lowest accuracy level for the weather class. Furthermore, these models are not useful in practice as they are able to correctly predict only one class. Among the other three models, the proposed ensemble algorithm can accurately predict animal, equipment, and weather classes, while the random forest algorithm has the highest accuracy in predicting the vegetation class.

It is also observed in all the three models that vegetation-related outages have the lowest percentage of accuracy. This fact might be related to two factors: first, the low number of samples of this class and second, the unpredictable nature of this kind of outage. Figure 6 shows the accuracy of each model

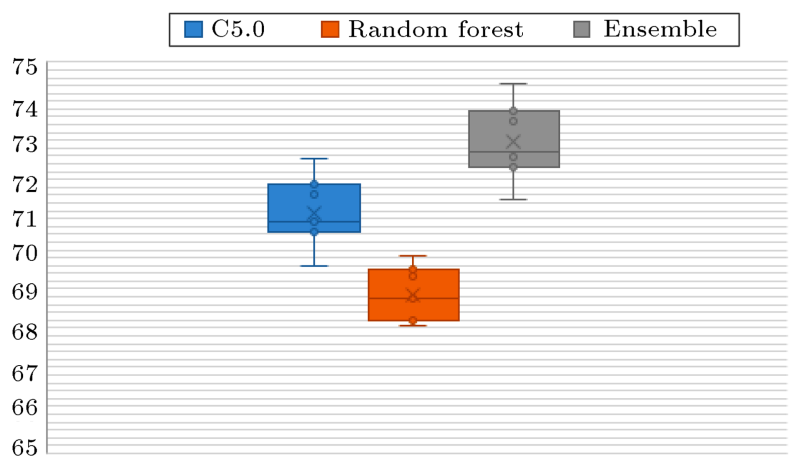

Figure 6. Comparison of the accuracy of predictors. with different train and test sets. It is observed that the proposed ensemble has the best performance and random forest is the least desirable model in terms of accuracy.

\section{Conclusion}

In this paper, animal-, equipment-, vegetation-, and weather-related outages in a power distribution system are discussed. First, four models were trained to analyze the root cause of outages upon occurrence. In the experiments, it was shown that unbalanced nature of data could be overcome using the appropriate cost matrix. It was also proved that the degree of importance of a given root cause could be reflected in the same matrix and these models could adapt to the requirements of the distribution system operator. For example, if animal-related failures were the most important class, the cost matrix could be customized so that this kind of outage would have the highest priority in the resulting model. To improve performance of the predictor, it was proposed to combine random forest and C5.0 based models to form an ensemble model. The results showed that accuracy of prediction might increase by $5 \%$ and $2 \%$ in the ensemble model in comparison with the random forest and C5.0 models, respectively. The proposed data-mining-based model may shorten the repair time and improve reliability of the distribution power system. 


\section{References}

1. Zheng, K., Chen, Q., Wang, Y., et al. "A novel combined data-driven approach for electricity theft detection", IEEE Trans. Ind. Informatics, 15(3), pp. 1809-1819 (2018).

2. Jokar, P., Arianpoo, N., and Leung, V.C.M. "Electricity theft detection in AMI using customers consumption patterns", IEEE Trans. Smart Grid, 7(1), pp. 216-226 (2016).

3. Singh, S.K., Bose, R., and Joshi, A. "PCA based electricity theft detection in advanced metering infrastructure", 7th Int. Conf. Pow. Syst. (ICPS), pp. 441445 (2017).

4. Venkatesh, A., Cokkinides, G., and Meliopoulos, A.P.S. "3D-visualization of power system data using triangulation and subdivision techniques", Proc. 42nd Annu. Hawaii Int. Conf. Syst. Sci. HICSS, pp. 1-8 (2009).

5. McMorran, A.W., Ault, G.W., and McDonald, J.R. "Solving data integration challenges for web-based geographical power system data visualization using CIM", IEEE Power Energy Soc. 2008 Gen. Meet. Convers. Deliv. Electr. Energy 21st Century, PES (2008).

6. Bank, J.N., Omitaomu, O.A., Fernandez, S.J., et al. "Visualization and classification of power system frequency data streams", ICDM Work. 2009 - IEEE Int. Conf. Data Min., pp. 650-655 (2009).

7. Brown, R.E., Electric Power Distribution Reliability, 2nd Edn., CRC press (2008).

8. Schroder, T. and Kuckshinrichs, W. "Value of lost load: An efficient economic indicator for power supply security? A literature review", Front. Energy Res., 3, p. $55(2015)$.

9. Eskandarpour, R. and Khodaei, A. "Machine learning based power grid outage prediction in response to extreme events", IEEE Trans. Power Syst., 32(4), pp. 3315-3316 (2017).

10. Wanik, D.W., Anagnostou, E.N., Hartman, B.M., et al. "Storm outage modeling for an electric distribution network in Northeastern USA", Nat. Hazards, 79(2), pp. 1359-1384 (2015).

11. Doostan, M. and Chowdhury, B.H. "Power distribution system fault cause analysis by using association rule mining", Electr. Power Syst. Res., 152, pp. 140147 (2017).

12. Cai, Y. and Chow, M.Y. "Cause-effect modeling and spatial-temporal simulation of power distribution fault events", IEEE Trans. Power Syst., 26(2), pp. 794-801 (2011).

13. Chow, M. and Taylor, L.S. "Analysis and prevention of animal-caused faults in power distribution systems", IEEE Trans. Power Deliv., 10(2), pp. 995-1001 (1995).
14. Warlyani, P., Jain, A., Thoke, A.S., et al. "Fault classification and faulty section identification in teed transmission circuits using ANN", Int. J. Comput. Electr. Eng., 3(6), pp. 807-811 (2012).

15. Xu, L., Chow, M.-Y., and Taylor, L.S. "Power distribution fault cause identification with imbalanced data using the data mining-based fuzzy classification E-Algorithm", IEEE Trans. Power Syst, 22(1), pp. 164-171 (2007).

16. Doostan, M. and Chowdhury, B.H. "Power distribution system equipment failure identification using machine learning algorithms", IEEE Pow. Energy Soc. Gen. Meet., pp. 1-5 (2018)

17. Doostan, M., Sohrabi, R., and Chowdhury, B. "A data-driven approach for predicting vegetation-related outages in power distribution systems", arXiv Prepr. arXiv1807.06180, pp. 1-11 (2018).

18. Breiman, L. "Random forests", Mach. Learn., 45(1), pp. 5-32 (2001).

19. Quinlan, J.R. "C5.0 data mining tool", RuleQuest Research, 63, p. 64 (1997).

20. Quinlan, J.R. "C 4.5: Programs for machine learning", Morgan Kaufmann Ser. Mach. Learn. San Mateo, CA Morgan Kaufmann (1993).

21. Hinton, G. and Van Camp, D. "Keeping neural networks simple by minimizing the description length of the weights", Proc. 6th Ann. ACM Conf. on Computational Learning Theory (1993).

22. Raspisaniye Pogodi Ltd, Reliable Prognosis. Weather and Climate Change, Available Online at 2019: https://rp5.ru/.

23. Liu, H. and Motoda, H. "Feature selection for knowledge discovery and data mining", 454, Springer Science \& Business Media (2012).

24. Guyon, I. and Elisseeff, A. "An introduction to variable and feature selection", J. Mach. Learn. Res., 3(Mar), pp. 1157-1182 (2003).

\section{Biographies}

Mohammad Sadegh Bashkari received the BSc and MSc degrees in Software Engineering from Shiraz University, Shiraz, Iran, in 2011 and 2013, respectively. He is currently a $\mathrm{PhD}$ candidate in Software Engineering at Shiraz University. His primary research interests are data analysis and application of data mining methods to interdisciplinary research areas.

Ashkan Sami obtained his BSc from Virginia Tech, USA, in 1991; MSc in AI and Robotics from Shiraz University, Iran, in 1996; and PhD from Tohoku University, Japan, in 2006. His PhD thesis became the main idea of a national project funded by the Japanese government and earned him a tenured faculty position at Tohoku University. Currently, as an associate professor in CSE and IT Department at Shiraz University, Dr. 
Sami conducts high quality interdisciplinary research on applied artificial intelligence, data mining, cyber security, and software engineering. He has graduated more than $100 \mathrm{MSc}$ and $\mathrm{PhD}$ students under his direct supervision, has served in various program committees of reputable national and international conferences, and is a technical committee member of IEEE Software Engineering and IEEE Industrial Electronics.

Mohammad Rastegar received the BSc, MSc, and PhD degrees from Sharif University of Technology, Tehran, Iran, in 2009, 2011, and 2015, respectively, all in Electrical Engineering. He is currently an Assistant
Professor with the School of Electrical and Computer Engineering at Shiraz University, Shiraz, Iran. His current research interests include modeling home energy management systems, plug-in hybrid electric vehicle operation, and power system reliability and resiliency studies.

Mohammad Javad Bordbari received the BSc and MSc degrees in Electrical Engineering from Shiraz University of Technology and Shiraz University in 2015 and 2018, respectively. His current research interests include modeling home energy management systems, energy efficiency analysis in buildings, and power system reliability and resiliency studies. 